\title{
Hepatocellular carcinoma (HCC) in a patient with cystic fibrosis and liver disease (cirrhosis)
}

Petros Pouliopoulos ${ }^{1}$, Katerina Manika ${ }^{1}$, Eleni Faniadou ${ }^{1}$, Stavros Galanis ${ }^{2}$, Konstantinos Vasileiou ${ }^{3}$, Maria Sionidou ${ }^{1}$, Antonios Avgerinos ${ }^{3}$, loannis Kioumis ${ }^{1}$

\begin{abstract}
Nowadays, cystic fibrosis is a disease in which, due to early diagnosis and advances in treatment, the age limit has been considerably extended, resulting in many patients living beyond the fourth decade of life. However, due to increased life expectancy, the risk of developing diseases due to chronic complications and pathophysiology of cystic fibrosis has increased. The case of a male patient with cystic fibrosis and documented liver disease related to the primary disease is described. When the annual liver ultrasound was performed, masses of a heterogeneous echostructure in the right lobe of the liver were visualized. A three-phase liver computed tomography followed, showing a space-occupying lesion, which ultimately proved to be a hepatocellular carcinoma. It is worth mentioning that while one of the rare complications of liver cystic fibrosis disease is cholangiocarcinoma, this patient developed a hepatocellular carcinoma.
\end{abstract}

\author{
AFFILIATION \\ 1 Department of Cystic Fibrosis, Pulmonary \\ Department, Aristotle University of \\ Thessaloniki, G. Papanikolaou General \\ Hospital, Thessaloniki, Greece \\ 2 Department of Radiology, G. Papanikolaou \\ General Hospital, Thessaloniki, Greece \\ 3 Gastroenterology Department, G. \\ Papanikolaou General Hospital, Thessaloniki, \\ Greece
}

\section{CORRESPONDENCE TO \\ Katerina Manika. Department of Cystic Fibrosis, Pulmonary Department, Aristotle University of Thessaloniki, G. Papanikolaou General Hospital, Thessaloniki, Greece. E-mail: ktmnO5@yahoo.gr}

\section{KEYWORDS}

cystic fibrosis-associated liver disease, hepatocellular carcinoma, cystic fibrosis

Received: 15 May 2021

Revised: 8 December 2021

Accepted: 24 December 2021

\section{INTRODUCTION}

Due to the prolonged survival of patients with cystic fibrosis, we are increasingly being called upon to face new challenges in dealing with the complications and development of complications associated with the primary disease ${ }^{1-3}$. This patient developed hepatocellular carcinoma, due to cirrhosis and portal hypertension, detected on his routine annual upper abdominal ultrasound. This finding underlies the significance of annual screening tests for people with cystic fibrosis.

\section{CASE PRESENTATION}

A 35-year-old patient with a history of cystic fibrosis since he was a neonate (meconium ileus) and E379X and W496X gene mutations, underwent an upper abdominal ultrasound as part of the follow-up of the known hepatic cirrhosis with concomitant portal hypertension. From the accompanying medical history, he had diabetes mellitus and was under insulin therapy, cirrhosis during adolescence, bronchiectases (Figure 1), and chronic pulmonary infection with Pseudomonas aeruginosa, while his respiratory function was as follows: Tiffeneau 60\%, FVC 2.52 L (53\%), FEV1 1.32 L (33\%).

The findings of the ultrasound were consistent with the already known cirrhosis; however, heterogeneous echostructures were shown in the right lobe of the liver, which required further investigation. A three-phase computed tomography of the liver followed, which showed a space occupying lesion measuring $13 \times 10 \times 6 \mathrm{~cm}$ on the anterior surface of the right lobe, showing an uptake of the

\section{Figure 1. Patient's chest x-ray}

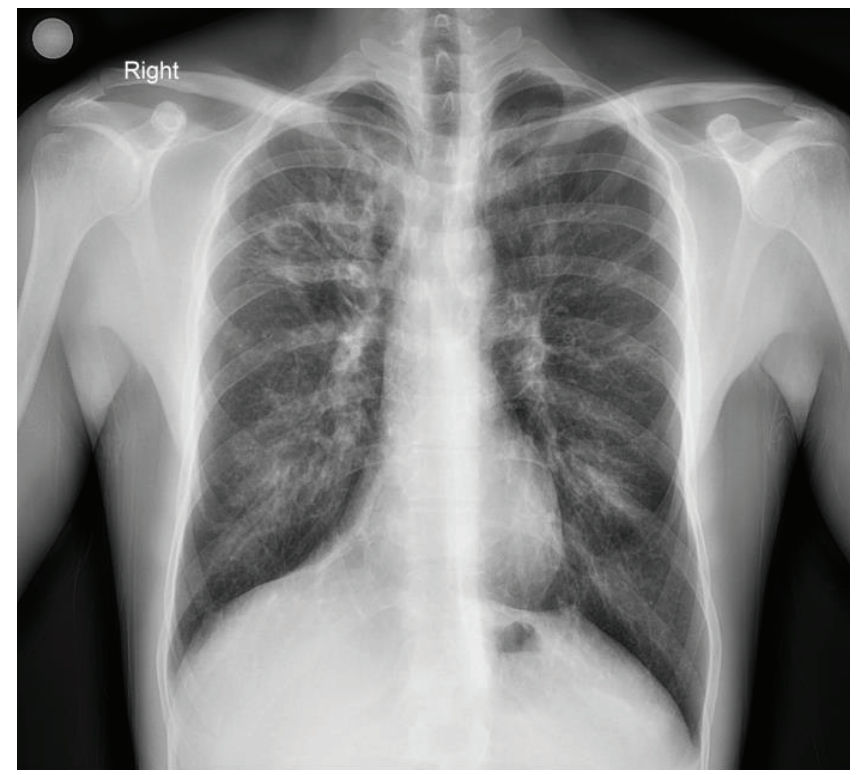


Figure 2. Images of a three-phase liver computed tomography. (A) A space occupying lesion measuring $13 \times 10 \times 6 \mathrm{~cm}$ is seen on the anterior surface of the right lobe of the liver, which uptakes the contrast agent in the arterial phase (B), followed by flushing in the portal (C) and delayed phase (D), a characteristic imaging finding that led to the diagnosis of a hepatocellular carcinoma

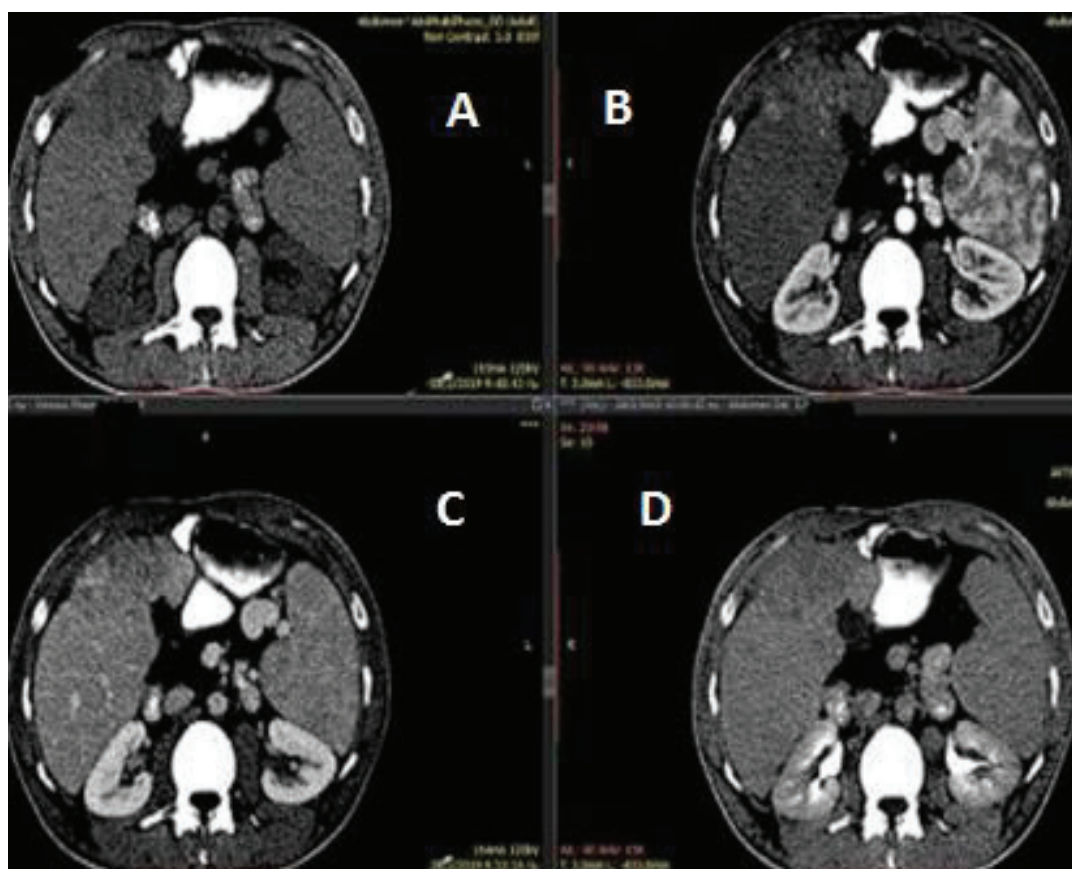

contrast agent in the arterial phase, followed by flushing of the contrast agent in the portal and delayed phase, a finding which was consistent with a hepatocellular carcinoma (Figure 2). A very high value of a-fetoprotein was initially found, at $227 \mathrm{ng} / \mathrm{mL}$ and at $665.73 \mathrm{ng} / \mathrm{mL}$ at a follow-up examination. Screening for hepatitis (B and $\mathrm{C}$ ), metabolic, or autoimmune disorders was negative, with no history of alcoholism. Subsequently, the patient underwent magnetic resonance imaging of the abdomen, which also revealed a lesion on the anterior surface of the right lobe of the liver, with confluent nodular satellite lesions.

Based on the above imaging findings and the particularly high value of a-fetoprotein, the diagnosis of a hepatocellular carcinoma was established. Chemoembolization was not possible, due to the size of the lesion, so hepatologists suggested the initiation of an antineoplastic agent (sorafenib).

\section{DISCUSSION}

Cystic fibrosis is an inherited disease that is transmitted through its autosomal recessive nature. It is caused by a variety of mutations in the cystic fibrosis transmembrane conductance regulator (CFTR) gene, located on chromosome 7. This gene encodes a regulatory protein (Cystic Fibrosis Transmembrane Conductance Regulator) which controls the passage of chlorine through the epithelial cell membranes of various organs of the body, such as the lungs, pancreas, liver, sweat glands, and intestine. Mutations in this gene cause reduced production or functionality of the protein, resulting in the epithelium of the affected organs producing viscous sticky mucus that obstructs the pores of the glands, resulting in progressive destruction of the organ tissue (fibrosis) and their eventual failure ${ }^{4,5}$.

With respect to hepatic involvement, mutations in the CFTR gene lead to abnormalities in composition, alkalinity, and bile flow, resulting in the production of visceral secretions in the bile ducts, which, in combination with the effect of cytotoxic or infectious agents, lead to their obstruction. Obstruction of the bile ducts subsequently leads to liver damage, resulting in the development of fibrosis, initially, and cirrhosis later on. However, the cause of the different severity of liver involvement among patients with cystic fibrosis has not been clarified. Many patients with cystic fibrosis have no clinical symptoms or signs of liver disease despite the existence of focal biliary cirrhosis ${ }^{4,5}$.

Fibrosis is thought to lead to cirrhosis over a period of years, a procedure which is usually asymptomatic ${ }^{2,4,5}$. The prevalence of liver disease in patients with CF ranges between $2 \%$ and $37 \%^{2,4}$. Combination of cirrhosis of the liver with portal hypertension occurs in approximately 10\% of patients with CFLD (Cystic Fibrosis Liver Disease) and peaks in adolescence ${ }^{2}$. The use of ursodeoxycholic acid improves the biochemical markers of CFLD and may slow down its development ${ }^{2,4}$. CFLD is almost always associated 
with pancreatic insufficiency ${ }^{5}$. One of the rare complications of liver cirrhosis in individuals with cystic fibrosis is the development of cholangiocarcinoma ${ }^{5}$. However, what is of interest in this patient case is the development of a hepatocellular carcinoma due to cirrhosis and portal hypertension, which is not related to the pathophysiology of the disease but is clearly associated to cirrhosis.

Hepatocellular carcinoma is the most common liver tumor (90-95\% of primary malignant liver tumors) and the number of new cases is increasing 2,6 . The major causative agents are chronic hepatitis $\mathrm{B}$ and $\mathrm{C}$ infections, which account for over $80 \%$ of cases worldwide ${ }^{6}$. Other etiologic factors are alcohol and smoking, liver adenoma and repeated ingestion of aflatoxin containing foods ${ }^{7}$. The incidence ratio between men and women is $3: 2^{2}$.

The patient presented here developed hepatocellular carcinoma due to cirrhosis and portal hypertension, with a negative history of hepatitis B, C and alcoholism. After reviewing the literature, at least 3 more patients with CFLD have developed hepatocellular carcinoma ${ }^{1-3}$. The first case is a 32-year-old pregnant woman with cystic fibrosis and CFLD who was diagnosed with a focal liver injury during the annual upper abdominal ultrasound. After undergoing a biopsy and the diagnosis of hepatocellular carcinoma was confirmed, she was finally treated with laser ${ }^{2}$. The second case is an 18-year-old woman who had symptoms of fever, hepatomegaly, and weight loss. Through investigation, a mass was found in the right lobe of the liver, that was initially treated as an abscess, and upon the persistently nonimproving picture, she underwent a biopsy, with a diagnosis of HCC. This patient died 4 days after the biopsy, with a severe clinical status ${ }^{1}$. The third case relates to a 34-yearold male patient CFLD, who was diagnosed with HCC after a biopsy of a lesion initially found during an annual abdominal ultrasound. The characteristic feature of this patient is that he had a normal a-fetoprotein (aFP) value with an increase of Ca19-9. He did not meet the liver transplant criteria, as his mass was of $7 \mathrm{~cm}$ and sorafenib was initiated, just like for our own patient ${ }^{3}$.

Additionally, what is of interest in our patient is that the diagnosis was performed on the basis of the imaging findings and the extremely high value of a-fetoprotein $(665.73 \mathrm{ng} / \mathrm{mL})$, as values greater than $400-500 \mathrm{ng} / \mathrm{mL}$ are considered diagnostic for HCC, without performing a liver biopsy6,8.

\section{CONCLUSION}

This is an uncommon case of hepatocellular carcinoma in a patient with cystic fibrosis. The annual examination led to an unexpected finding, which highlights the need for more regular screening of patients with cystic fibrosis and hepatic insufficiency, perhaps twice a year, with an abdominal ultrasound and possibly measuring a-fetoprotein, for early diagnosis of an eventual malignancy. As the survival of patients with cystic fibrosis grows, so do previously unknown manifestations and complications of the disease, involving a wide range of medical specialties.

\section{CONFLICTS OF INTEREST}

The authors have completed and submitted the ICMJE Form for Disclosure of Potential Conflicts of Interest and none was reported.

\section{FUNDING}

There was no source of funding for this research.

\section{ETHICAL APPROVAL AND INFORMED CONSENT}

Ethical approval was not required for this study. Verbal informed consent was provided by the patient's wife.

\section{DATA AVAILABILITY}

The data supporting this research can not be made available for privacy reasons.

\section{PROVENANCE AND PEER REVIEW}

Not commissioned; externally peer reviewed.

\section{REFERENCES}

1. Kelleher T, Staunton M, O'Mahony S, McCormick PA. Advanced hepatocellular carcinoma associated with cystic fibrosis. Eur J Gastroenterol Hepatol. 2005;17(10):11231124. doi:10.1097/00042737-200510000-00018

2. Mckeon D, Day A, Parmar J, Alexander G, Bilton D. Hepatocellular carcinoma in association with cirrhosis in a patient with cystic fibrosis. J Cyst Fibros. 2004;3(3):193195. doi:10.1016/j.jcf.2004.04.006

3. O'Donnell DH, Ryan R, Hayes B, Fennelly D, Gibney RG. Hepatocellular carcinoma complicating cystic fibrosis related liver disease. J Cyst Fibros. 2009;8(4):288-290. doi:10.1016/j.jcf.2009.05.002

4. Kobelska-Dubiel N, Klincewicz B, Cichy W. Liver disease in cystic fibrosis. Prz Gastroenterol. 2014;9(3):136-141. doi:10.5114/pg.2014.43574

5. Perdue DG, Cass OW, Milla C, et al. Hepatolithiasis and cholangiocarcinoma in cystic fibrosis: A case series and review of the literature. Dig Dis Sci. 2007;52(10):26382642. doi:10.1007/s10620-006-9259-1

6. Trevisani F, D'Intino PE, Morselli-Labate AM, et al. Serum a-fetoprotein for diagnosis of hepatocellular carcinoma in patients with chronic liver disease: Influence of $\mathrm{HBsAg}$ and anti-HCV status. J Hepatol. 2001;34(4):570-575. doi:10.1016/S0168-8278(00)00053-2

7. Erkekoglu P, Oral D, Chao M-W, Kocer-Gumusel B. Hepatocellular Carcinoma and Possible Chemical and Biological Causes: A Review. J Environ Pathol Toxicol Oncol. 2017;36(2):171-190. doi:10.1615/JEnvironPatholToxicolOncol.2017020927

8. Murugavel KG, Mathews S, Jayanthi V, et al. Alpha-fetoprotein as a tumor marker in hepatocellular carcinoma: investigations 
in south Indian subjects with hepatotropic virus and aflatoxin etiologies. Int J Infect Dis. 2008;12(6):e71-e76.

doi:10.1016/j.ijid.2008.04.010 